\title{
Dielectric Normal Mode Process in Semidilute and Concentrated Solutions of Poly(2,6-dichloro-1,4-phenylene oxide)
}

\author{
Keiichiro ADACHI and Tadao KOTAKA \\ Department of Macromolecular Science, Faculty of Science, \\ Osaka University, Toyonaka, Osaka 560, Japan
}

(Received September 11, 1985)

\begin{abstract}
The dielectric normal mode process was investigated on semi-dilute and concentrated solutions of poly(2,6-dichloro-1,4-phenylene oxide) (PDCPO-M) in a good solvent, chlorobenzene, at $300 \mathrm{~K}$. Four linear, short chain samples with molecular weight $M$ less than $20 \times 10^{3}$, two nearly linear samples of intermediate $M$, and a high $M$, randomly branched sample (PDCPO-380) of $M=380 \times 10^{3}$ were used. The dielectric relaxation time $\tau$ for $40 \mathrm{wt} \%$ solutions of the linear samples was approximately proportional to $M^{2}$, indicating Rouse behavior of nonentangled chains. The loss curves of the PDCPO-17 solutions were symmetrical in shape, and the half width $\Delta$ increased slightly with increasing concentration $C$ due presumably to the increase in the local friction constant $\zeta$. On the other hand, for the branched, high $M$ PDCPO-380 sample, its loss curve not only became broader with increasing $C$, but split into two peaks at about $C=30 \mathrm{wt} \%$. This is presumably because the relaxation time of longer branches of the sample were retarded more strongly by entanglement. From the relaxation strength, the mean square end-to-end distance $\left\langle r^{2}\right\rangle$ was determined as a function of $C$ for PDCPO-17 solutions. The slope of the $\log \left\langle r^{2}\right\rangle v s$. $\log C$ plot was approximately -0.08 in the range of $0.2<C<1.0$. This value is much smaller than -0.25 expected from the scaling theory. This discrepancy may be attributed to the weak excluded volume effect for the low molecular weight sample.
\end{abstract}

KEY WORDS Poly(2,6-dichloro-1,4-phenylene oxide) / Dielectric Normal Mode Process / Dielectric Relaxation Time / Dipole Moment / End-to-End Vector / Bead and Spring Model / Tube Model / Entanglement / Branched Polymer /

Recently, we reported a series of dielectric studies on "type-A" polymers" having the component of the dipole moment parallel to the chain contour. ${ }^{2-7}$ The dielectric normal mode process due to the fluctuation of the endto-end vector in such polymers was first studied by Stockmayer and his coworkers ${ }^{8-10}$ and later by some others. ${ }^{11-13}$ However, these works were concerned only with the dynamics of non-entangled chains described by a beadspring model of Rouse $^{14}$ and Zimm. ${ }^{15}$ In contrast, we explored two new features of the normal mode process in the dynamic and static aspects which were not studied by these authors. They are: (1) the effects of entanglement on the relaxation time and its distribution and
(2) the relationship between the relaxation strength and the mean square end-to-end distance $\left\langle r^{2}\right\rangle$ of the polymer.

In the series of studies, we investigated the effects of entanglement on the normal mode process for bulk ${ }^{3,5}$ and solutions ${ }^{7}$ of relatively narrow distribution cis-polyisoprene (cis-PI). The dependence of the relaxation time on molecular weight $M$ and concentration $C$ agreed well with the dynamic scaling theory based on the tube model by de Gennes ${ }^{16-18}$ and Doi and Edwards. ${ }^{19}$ The distribution of the relaxation time became broader and more skewed with increasing $M$ and $C$ in a systematic manner, which was also explained satisfactorily in terms of the tube model. ${ }^{5,7}$ 
Almost 20 years ago, $\mathrm{Zimm}^{15}$ predicted that the dielectric relaxation strength $\Delta \varepsilon$ of the normal mode process of a type-A polymer is proportional to the mean square endto-end distance $\left\langle r^{2}\right\rangle .^{20,21}$ However, this relation had never been tested to determine $\left\langle r^{2}\right\rangle$ until our recent study. ${ }^{7}$ We found for solutions of poly(2,6-dichloro-1,4-phenylene oxide) (PDCPO) from the dependence of $\Delta \varepsilon$ on polarity of solvents that the ratio of the internal to external field involved in the normal mode process is close to unity. ${ }^{6}$ Based on this result, we determined the $C$ dependence of $\left\langle r^{2}\right\rangle$ from $\Delta \varepsilon$ of the normal mode process for semidilute and concentrated solutions of cis-PI in benzene. $^{7}$ The results of $\left\langle r^{2}\right\rangle v s$. $C$ could not be explained unequivocally by any of the existing theories, though they agreed approximately with the scaling theory by Daoud and Jannink $^{22}$ and the mean field theories by Edwards and his coworkers. ${ }^{23.24}$

We extended these studies to semidilute and concentrated solutions of PDCPO. Previously, we reported dielectric data of the dilute solutions in chlorobenzene $(\mathrm{ClBz}),{ }^{2}$ for which we concluded that the low molecular weight samples of PDCPO with $M<2 \times 10^{4}$ are linear, but the samples in the range of $M>2 \times 10^{4}$, randomly branched. ${ }^{2}$ In this study, we examined both the linear, low $M$ and randomly branched, high $M$ PDCPO samples in semidilute and concentrated solutions.

\section{EXPERIMENTAL}

\section{Materials}

Some of the PDCPO samples used were the same as reported previously. ${ }^{2}$ Others were fractions of previous samples. Details of the preparation and characterization were reported previously. ${ }^{2}$ The characteristics of the samples are given in Table I. The samples are coded as PDCPO-M with $M$ being the value of the weight average molecular weight represented in the unit of 1000 . The solvent, $\mathrm{ClBz}$, was purified by distillation with calcium
Table I. Sample characteristics

\begin{tabular}{ccc}
\hline Code & $10^{-3} M_{w}$ & $M_{w} / M_{n}$ \\
\hline PDCPO-07 & 6.8 & 1.18 \\
PDCPO-08 & 8.3 & 1.18 \\
PDCPO-14 & 13.5 & 1.15 \\
PDCPO-17 & 16.6 & 1.26 \\
PDCPO-25 & 25.1 & 1.29 \\
PDCPO-45 & 45.0 & 1.47 \\
PDCPO-380 & 380 & 1.50 \\
\hline
\end{tabular}

hydride.

\section{Methods}

Dielectric measurement was carried out in the range of frequency $f$ from $3 \mathrm{~Hz}$ to $100 \mathrm{MHz}$. The bridges and capacitance cell were described previously. ${ }^{2,3}$ In the present solutions, relatively high ionic conductivity $g_{0}$ was observed. In order to subtract the contribution of $g_{0}$ from the dielectric loss $\varepsilon^{\prime \prime}$, we estimated $g_{0}$ by extrapolating the plot of a.c. conductivity $g(f) v s . f^{2}$ to zero frequency as proposed by Jones et al. ${ }^{10}$ Thus, $g(f)-g_{0}$ was converted to $\varepsilon^{\prime \prime}$. Measurement of the density $\rho$ was also described previously. ${ }^{7}$ For the $\mathrm{ClBz}$ solutions of PDCPO, we determined $\rho$ at $300 \mathrm{~K}$ as

$$
\rho=1.08+1.31 w-0.83 w^{2}
$$

where $w$ denotes the weight fraction of the polymer.

\section{RESULTS AND DISCUSSION}

\section{Behavior of Linear Short Chain PDCPO}

Figure 1 shows the $\varepsilon^{\prime \prime}$ curves at $300 \mathrm{~K}$ for PDCPO-17 solutions with varying $C$. The dashed curve indicates the loss curve for 2.5 $\mathrm{wt} \%$ solution reported previously. ${ }^{2} \mathrm{We}$ recognize that with increasing $C$, the loss maximum frequency $f_{\mathrm{m}}$ shifts to the low frequency side, and at the same time, the half width $\Delta$ of the loss peak increases. These tendencies of decreasing $f_{\mathrm{m}}$ and broadening of the loss curve with increasing $C$ are generally the case for 


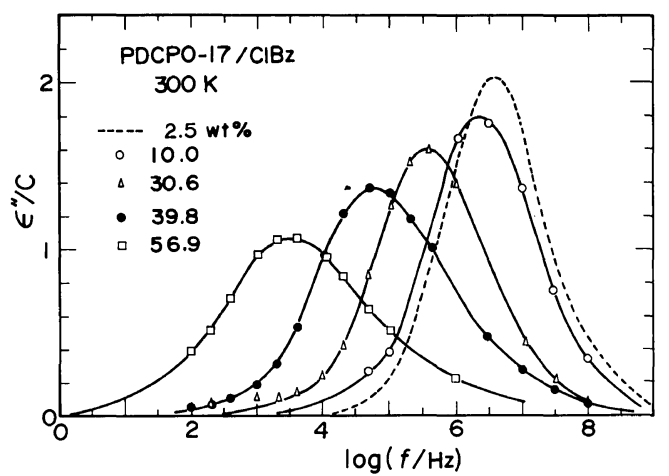

Figure 1. Frequency dependence of the dielectric loss factor $\varepsilon^{\prime \prime}$ divided by concentration $C$ (in $\mathrm{g} \mathrm{cm}^{-3}$ ) for chlorobenzene solutions of PDCPO- 17 at $300 \mathrm{~K}$. The polymer concentrations of the solutions are given in the figure in $\mathrm{wt} \%$.

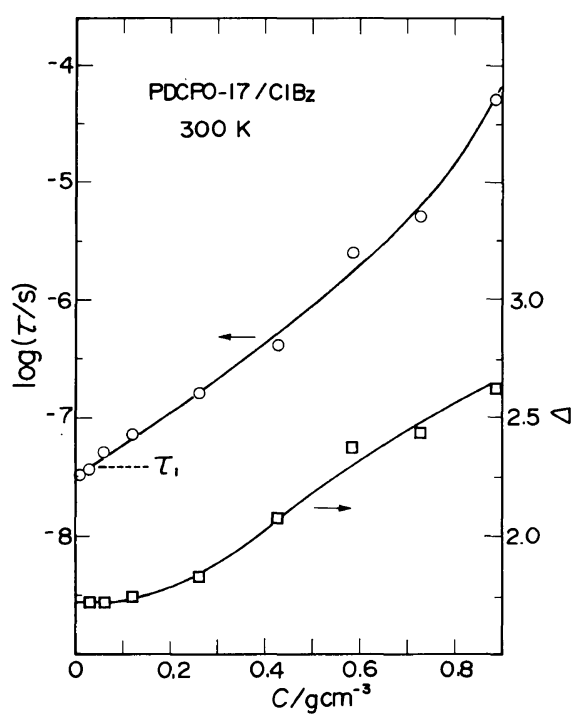

Figure 2. Concentration $C$ dependence of the relaxation time $\tau$ and the half width $\Delta$ of the loss curve (in the unit of decade) for chlorobenzene solutions of PDCPO-17.

solutions of all linear-chain type-A polymers including high $M$ cis-PI. However, their quantitative behavior was different, and presumably the underlying molecular mechanisms should be different between the linear short chain PDCPO and high $M$ cis-PI solutions, as will be discussed later.

Figure 2 shows the $C$ dependence of the

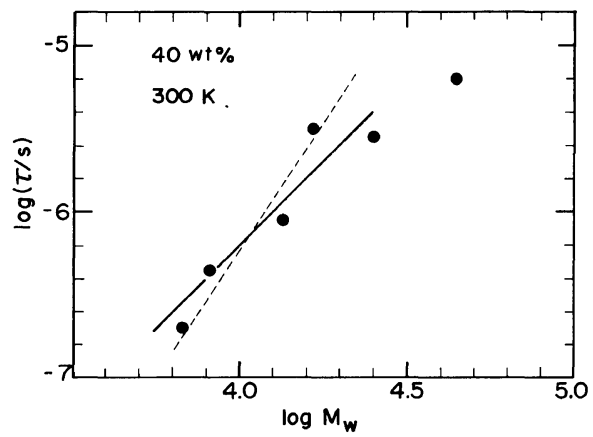

Figure 3. Molecular weight $M_{w}$ dependence of the relaxation time $\tau$ for solutions of PDCPO-M at the concentration of $40 \mathrm{wt} \%$. The full and dashed lines indicate the slope of 2 and 3, respectively.

logarithm of the relaxation time $\tau\left(=1 / 2 \pi f_{\mathrm{m}}\right)$ and the half width $\Delta$ of the loss curve for the PDCPO-17 solutions. The Rouse-Zimm theory ${ }^{14.15}$ predicts the longest relaxation time $\tau_{1}$ of dilute solutions as

$$
\tau_{1}=2 M \eta_{\mathrm{s}}[\eta] /\left(0.586 R T \lambda_{1}^{\prime}\right)
$$

for the non-draining model, where $\eta_{\mathrm{s}},[\eta]$, and $\lambda_{1}^{\prime}$ represent the solvent viscosity, the intrinsic viscosity, and the first eigenvalue $(=4.04)$, respectively. The dashed line in Figure 2 indicates the value of $\tau_{1}$ calculated by eq 2 . The theory predicts, at least, the value of $\tau_{1}$ of the right order of magnitude.

In Figure 2, we recognize that $\log \tau$ of this low $M$ PDCPO-17 sample is nearly directly proportional to $\mathrm{C}$ over a considerably wide range of $C(<0.7)$. Thus, $\log \tau$ may be expressed as:

$$
\log \tau=-7.50+2.48 C+0.864 C^{2}
$$

The half width $\Delta$ is almost independent of $C$ in the range of $\log C<-0.12$, while in the range of higher $C$, it increases gradually with $C$. As seen in Figure 1, the broadening of the loss curve with increasing $C$ is symmetrical. This behavior is in contrast to that in solutions of high $M$, linear-chain cis-PI. For the cis-PI system, we observed that with increasing $C$, the loss curve shifts more rapidly toward the low frequency side than the present system, 
and at the same time, it broadens asymmetrically. $^{7}$

Figure 3 shows the $M$ dependence of the relaxation time $\tau$ for $40 \mathrm{wt} \%$ solutions of linear PDCPO with $M<20 \times 10^{3}$ and those of the slightly branched PDCPO with $20 \times 10^{3}<$ $M<45 \times 10^{3}$. The full and dashed lines indicate slopes 2 and 3, respectively, corresponding to the theoretical values for non-entangled and entangled systems of linear chains. ${ }^{16,19}$ Since the data points are scattered, it is difficult to determine the slope precisely. However, we recognize that slope 2 appears to be more reasonable, since we expect that the chains are not entangled with each other in the present systems.

To discuss the $C$ and $M$ dependences of $\tau$ for the normal mode process, we need to know the molecular weight, $M_{\mathrm{e}}$, between entanglements or the characteristic molecular weight $M_{\mathrm{c}}$ $\left(=2 M_{\mathrm{e}}\right),{ }^{5.7}$ beyond which the molecules supposedly become entangled with one another. Unfortunately, $M_{\mathrm{e}}$ (or $M_{\mathrm{c}}$ ) for PDCPO has not been reported. Donald and $\mathrm{Kramer}^{25}$ estimated $M_{\mathrm{e}}$ of bulk poly(2,6-dimethyl-1,4phenylene oxide) (PDMPO) to be 7000 from fracture experiments on blends of PDMPO and polystyrene. From the similarity of the molecular structure of PDCPO to PDMPO, we may estimate $M_{\mathrm{e} 0}$ of bulk PDCPO to be 9400 by taking only the ratio of molecular weights of the repeat units into account. It is known that $M_{\mathrm{e}}$ is proportional to $C^{-1}$ in concentrated solutions ${ }^{26.27}$ and to $C^{-1.5}$ in the semidilute region in a good solvent. Since $M$ of PDCPO-17 is less than $M_{\mathrm{c} 0}(=18800)$ of PDCPO in the bulk state estimated above, the molecules of PDCPO-17 are not entangled in the entire range of $C$ examined in this study. Thus, the dependences of $\tau$ and $\Delta$ on $C$ of this sample shown in Figure 2 should reflect the features of non-entangled, linear PDCPO chains.

In $40 \mathrm{wt} \%$ solutions, $M_{\mathrm{c}}\left(\simeq M_{\mathrm{co}} / 0.40\right)$ is estimated to be $47 \times 10^{3}$. Thus, all PDCPO molecules shown in Figure 3 are not entangled because $M<M_{\mathrm{c}}$. This explains the $M^{2}$ dependence of $\tau$ shown in Figure 3, instead of the well known $M^{3.5}$. dependence of entangled linear chain systems. ${ }^{26,27}$

Generally, $\tau$ for non-entangling, short linear chains in condensed systems may be best approximated by the free draining model of Rouse: ${ }^{14}$

$$
\tau=\zeta N^{2} b^{2} /\left(3 \pi^{2} k_{\mathrm{B}} T\right)
$$

where $\zeta, N$, and $b$ denote the friction constant per bead, the number of beads in a chain and the average distance between the beads, respectively. Since the excluded volume effect is weak in the present system because of its low molecular weight, we may neglect the small $C$ dependence of $N b^{2}\left(=\left\langle r^{2}\right\rangle\right)$ in a good solvent. Thus, the $C$ dependence of $\tau$ shown in Figure 2 and by eq 3 may be attributed to the $C$ dependence of $\zeta$. Then, using eq 3 , we may cast the $C$ dependence of $\log \zeta$ in the form:

$$
\log \left(\zeta / \zeta_{0}\right)=\alpha_{1} C+\alpha_{2} C^{2}
$$

where $\alpha_{1}(=2.48)$ and $\alpha_{2}(=0.864)$ are constants, and $\zeta_{0}$ is the friction constant per bead at $C=0$, which can be calculated with the data of $\eta_{\mathrm{s}}$ and $[\eta]$ based on the Rouse-Zimm theory. ${ }^{14,15}$ de Gennes ${ }^{18}$ expressed the longest relaxation time in a semidilute solution by assuming $\zeta$ is independent of $C$. Equation 5 suggests that this assumption is not satisfactory, since $\tau$ of non-entangling chains can vary with $C$.

The $C$ dependence of $\zeta$ was discussed by Ferry, based on the free volume theory. ${ }^{27}$ The left hand side of eq 5 corresponds to the concentration shift factor $\log a_{\mathrm{c}}$ used by Ferry to analyze rheological data. ${ }^{27.28}$ Moore and Ferry $^{28}$ determined $\log a_{\mathrm{c}}$ for polyisobutylene/ $n$-hexadecane system, and found that $\log a_{\mathrm{c}}$ is almost directly proportional to $C$ up to $C$ as high as 0.7 . They successfully explained, in terms of the free volume theory, the observed $C$ dependence of $\log a_{\mathrm{c}}$ over the whole range of $C$. The present results on $\log \left(\zeta / \zeta_{0}\right)$ vs. $C$ appear to be qualitatively 
similar to those of $\log a_{\mathrm{c}}$ reported by Ferry.

Here, probably, a brief comment on the shape of the loss curve and the $C$ dependence of the half width $\Delta$ of PDCPO-17 solutions should be given. The asymmetrical shape of the loss curve for the solutions of the high $M$ cis-PI is obviously due to the entanglement effect as discussed before. ${ }^{7}$ On the other hand. for unentangled systems, $\tau$ may depend on $C$ only through $\zeta$ and $\left\langle r^{2}\right\rangle$, according to eq 4 . However, since $\left\langle r^{2}\right\rangle$ is little dependent on $C$ in the present short-chain systems, the $C$ dependence of $\tau$ should come from that of $\zeta$. Then, by increasing $C, f_{\mathrm{m}}$ should shift to the low frequency side without changing $\Delta$, because $\zeta$ increases with $C$. However, there is the question as to why the symmetrical broadening of the loss curve should occur for the present PDCPO-17 system. We tentatively consider that the distribution of the relaxation time should have arisen from the spacially inhomogeneous $\zeta$ which increases with $C$. At present, there are no theories available to explain such behavior quantitatively. Another possibility might be that the mechanisms not taken into account in the bead-spring model such as cooperative motions of the chains might cause distribution of the relaxation time.

\section{Relaxation Behavior in Solutions of Branched $P D C P O$}

Figure 4 shows the $\varepsilon^{\prime}$ and $\varepsilon^{\prime \prime}$ curves for solutions of high $M$, branched PDCPO-380 in ClBz. With increasing $C$, the single peak of the loss curve splits into two peaks at about $C=30 \mathrm{wt} \%$ as is seen in this figure. This anomalous behavior must arise from the randomly branched structure of the sample, since such behavior has never been observed in solutions of linear polymers. In the $10 \%$ solution, $\varepsilon^{\prime}$ increased below $1 \mathrm{kHz}$. We attribute this to surface polarization as reported previously for dilute solutions of PDCPO. ${ }^{2}$ In solutions of higher concentration, the increase in $\varepsilon^{\prime}$ in this frequency region may be due to both the dielectric dispersion and surface polarization.

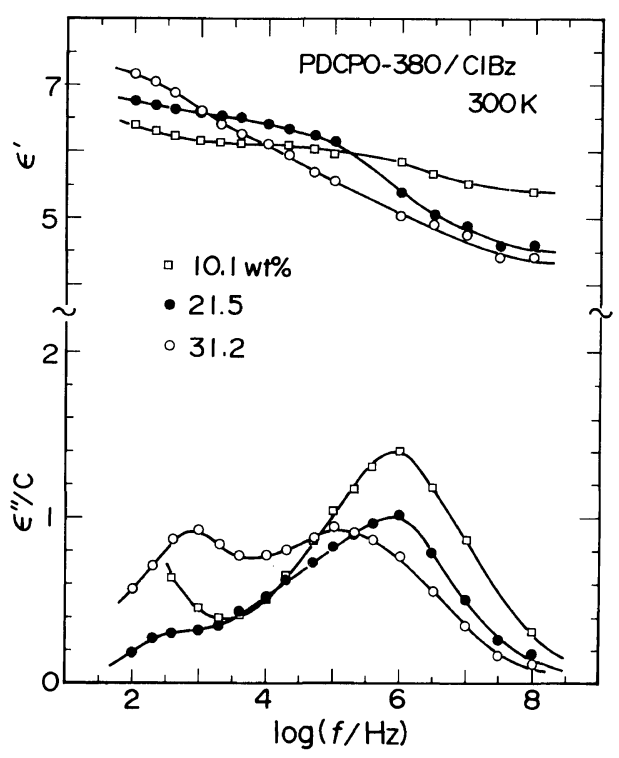

Figure 4. Frequency dependences of the dielectric constant $\varepsilon^{\prime}$ and loss factor $\varepsilon^{\prime \prime}$ divided by concentration $C$ (in $\mathrm{g} \mathrm{cm}^{-3}$ ) for chlorobenzene solutions of PDCPO-380 at $300 \mathrm{~K}$.

At present, it is difficult to estimate them separately.

As noted previously, PDCPO-380 molecules have about 19 branches on the average. ${ }^{2}$ Since the branching is considered to occur randomly, the sample should have a distribution of molecular weight $M_{\mathrm{b}}$ and the number of branches in the molecules. It was shown ${ }^{2,6}$ that the dielectric polarization of such a branched molecule is described by the sum of the polarizations of each branch. The relaxation time $\tau_{b}$ of the end-to-end fluctuation of a branch in dilute solution may be the same (or only slightly longer because one end is attached to the other chain) as the free linear chain having the same molecular weight. ${ }^{9}$ Hence, the distribution in $M_{\mathrm{b}}$ causes a distribution of the relaxation time. ${ }^{2}$ Assuming logarithmic normal distribution of the branch length, we determined the $M_{\mathrm{b} w} / M_{\mathrm{b} n}$ ratio to be 8.0 for PDCPO-380. ${ }^{2}$ Here, $M_{\mathrm{b} w}$ and $M_{\mathrm{b} n}$ denote the weight and number averages of $M_{\mathrm{b}}$.

It is expected that the dielectric relaxation spectrum of such a high $M$, highly branched 
molecule (having a broad distribution in the branches) in concentrated solution should be influenced strongly by entanglement effects. As discussed above, the $C$ dependence of $M_{\mathrm{c}}$ of a solution is approximately given by $M_{\mathrm{c}}=M_{\mathrm{co}} / C$. If we employ $M_{\mathrm{c} 0}=18800$ for PDCPO, we expect that branches with $M_{\mathrm{b}}>51 \times 10^{3}$ would become entangled in the $31 \mathrm{wt} \%\left(C=0.37 \mathrm{~g} \mathrm{~cm}^{-3}\right)$ solution. However, in the $10 \mathrm{wt} \%$ solution almost all branches of the PDCPO-380 molecules are not entangled, since the molecules rarely contain branches of $M_{\mathrm{b}}>190 \times 10^{3}$.

With increasing $C, \tau_{\mathrm{b}}$ of the non-entangled, short branches increase only slightly due to the increase in $\zeta$, but $\tau_{\mathbf{b}}$ for the long branches are prolonged due not only to the increase in $\zeta$ but to the particularly severe constraint by entanglement on the longer branches. The prolonged relaxation time of the end-to-end reorientation of entangled branches was proposed by de Gennes, ${ }^{29}$ and Doi and Kuzuu, ${ }^{30}$ for star-shaped polymers:

$$
\tau_{\mathrm{b}}=\tau_{\mathrm{d}} \exp \left(K M_{\mathrm{b}} / M_{\mathrm{e}}\right)
$$

where $\tau_{\mathrm{d}}$ is the relaxation time for the tube disengagement (reptation) process for a linear molecule having the same $M$ as the branch, and $K$ is a constant approximately $0.66 .^{30}$ This equation indicates that the relaxation time of the longer branches are strongly damped with increasing $C$, as they begin to entangle each other. In solution, only long branches with $M>M_{\mathrm{co}} / C$ are expected to be entangled, but short branches are not. Obviously, this causes splitting of the loss curve in the present PDCPO-380 solutions of $31 \mathrm{wt} \%$, but does not in the $10 \mathrm{wt} \%$ solution.

We compare in Figure 5 the loss curves of the 10.1 and $31.2 \mathrm{wt} \%$ solutions of PDCPO380 in an iso-friction constant state attained by shifting the latter to the high frequency side by $\log a_{\mathrm{c}}=0.98$ evaluated by using eq 4 . The $\varepsilon^{\prime \prime}$ curve (B) observed for the $31.2 \%$ solution is roughly resolved into two peaks: namely the curve (C) due to the non-entangled branches

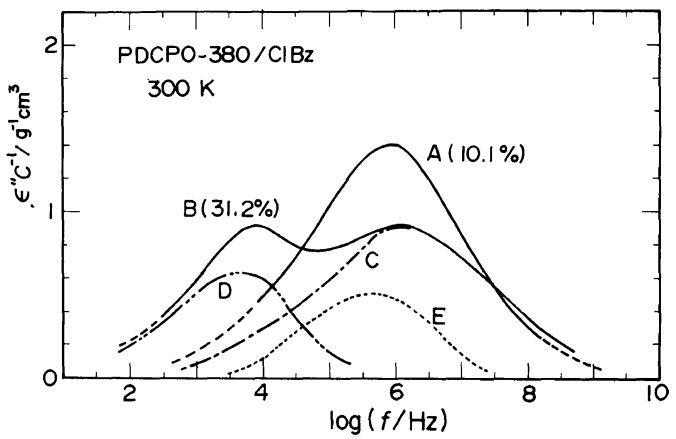

Figure 5. Comparison of the loss curves for 10.1 and $31.2 \mathrm{wt} \%$ solutions at an iso-friction constant state. (A) $\varepsilon^{\prime \prime}$ curve for the $10.1 \%$ solution at $300 \mathrm{~K}$. (B) $\varepsilon^{\prime \prime}$ curve for the $31.2 \%$ solution being shifted to the high frequency side so that the value of $\zeta$ is the same as the $10.1 \%$ solution. (C) A component of the curve (B) resolved. (D) Another component of the curve (B) resolved. (E) The curve (A) subtracted by (C).

and the curve (D) due to the entangled branches. The curve $(\mathrm{E})$ is drawn by subtracting the curve $(\mathrm{C})$ from the loss curve (A) observed for the $10.1 \%$ solution. Thus, the curve E corresponds to the $\varepsilon^{\prime \prime}$ curve for relatively long but not-yet-entangled branches in solution. By increasing $C$ from 10.1 to $31.2 \%$, the loss curve for the long branches shifts from (E) to (D). If we assume that the average $M_{\mathrm{b}}$ for the curves $C$ and $D$ are $c a .20 \times 10^{3}$ and $100 \times 10^{3}$, respectively, the magnitude of the shift from (E) to (D) is estimated to be 2.3 decades by using eq 4 and 6 . This approximately agrees with the results shown in Figure 5. It should be noted that since $M_{\mathrm{b}}$ is distributed continuously, the magnitude of the shift of $f_{\mathrm{m}}$ should be calculated for each branch from the distribution function of $M_{\mathrm{b}}$. However, we cannot further analyze the present system because of the lack of detailed information on the branch structure.

\section{Concentration Dependence of Relaxation Strength of PDCPO-17}

The relaxation strength $\Delta \varepsilon$ for solutions of PDCPO-17 was calculated from the area of the loss curve. Figure 6 shows the $C$ dependence of $\Delta \varepsilon$ divided by $C$. Theoretically $\Delta \varepsilon$ is written as 


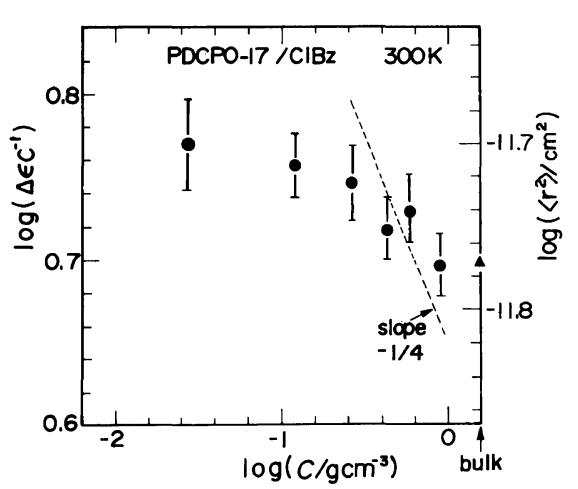

Figure 6. Concentration $C$ (in $\mathrm{gcm}^{-3}$ ) dependence of the relaxation strength $\Delta \varepsilon$ divided by $C$ and the mean square end-to-end distance $\left\langle r^{2}\right\rangle$ calculated with eq 7 . The dashed line represents the slope of $\log \left\langle r^{2}\right\rangle v s . \log C$ plot predicted by the scaling theory.

$$
\frac{\Delta \varepsilon}{C}=\frac{4 \pi N_{\mathrm{A}} \mu^{2}\left\langle r^{2}\right\rangle}{3 k_{\mathrm{B}} T M}
$$

where $N_{\mathrm{A}}$ is the Avogadro constant; $\mu$, the dipole moment per unit contour length; and $\left\langle r^{2}\right\rangle$, the mean square end-to-end distance. For the segmental mode in a solution of a polar molecule in a nonpolar liquid or in a polar liquid, the Lorentz-Lorenz field or the Onsager field approximation is often employed for the ratio of the internal to external field $F{ }^{31}$ However, we have shown that the ratio $F$ is close to unity for the normal mode process, and therefore eq 7 does not include this factor $F^{6}$

Using the value of $\mu\left(=2.85 \times 10^{-11} \mathrm{cgs}\right.$ esu $)$ of PDCPO estimated previously, ${ }^{2}$ we determined $\left\langle r^{2}\right\rangle$ from $\Delta \varepsilon / C$ and plotted it as shown in Figure 6. The value of $\left\langle r^{2}\right\rangle$ in a mixed solvent of $\mathrm{ClBz}-1$-bromohexane $(60: 40)$ was considered to be close to that in an unperterbed state. ${ }^{6}$ Therefore, $\left\langle r^{2}\right\rangle$ of PDCPO-17 (Code P9 in ref 6) in the mixed solvent is used as $\left\langle r^{2}\right\rangle$ in the bulk state. There have been many theoretical studies on the $C$ dependence of the coil dimension. ${ }^{17,22-24}$ However, only a few experimental studies have been conducted to check the validity of the theories mostly by the small angle neutron scattering (SANS) method. ${ }^{32-34}$ Previously we examined the $C$ dependence of $\left\langle r^{2}\right\rangle$ for $c i s$-PI/benzene systems over a wide range of $C$, and compared it with the scaling theory ${ }^{17,22}$ and the mean field theories. ${ }^{23,24}$ To determine coil dimensions, the conventional light scattering method is applicable only to dilute solutions, while SANS is applicable to condensed systems but requires huge facilities of a neutron source not readily accessible to us. Furthermore, the scattering methods allow us to determine only the mean square radius of gyration but not $\left\langle r^{2}\right\rangle$.

The critical concentration $C^{*}$ at which random coils in a solution begin to overlap is estimated to be $0.036\left(\log C^{*}=-1.44\right)$. According to the scaling theory by Daoud and Jannink, ${ }^{22}$ the $C$ dependence of $\left\langle r^{2}\right\rangle$ in the semidilute region $\left(C>C^{*}\right)$ is given by

$$
\left\langle r^{2}\right\rangle \propto C^{-(2 v-1) /(3 v-1)}
$$

where $v$ is the exponent for the $M$ dependence of $\left\langle r^{2}\right\rangle^{1 / 2}$ in dilute solution written as $\left\langle r^{2}\right\rangle^{1 / 2} \propto M^{v}$. In a good solvent, $v$ is usually taken to be $3 / 5$, and hence $\left\langle r^{2}\right\rangle$ varies in proportion to $C^{-1 / 4}$ in the good solvent. In Figure 6 , we recognize that the slope of $\log \left\langle r^{2}\right\rangle v s . \log C$ is smaller than the prediction of the scaling law. Since $M$ of the PDCPO-17 sample is relatively low, the blob model might not be a good approximation. It is also pointed out that $v=3 / 5$ is valid only for a very good solvent system at the high molecular weight limit. For low molecular weight samples, $v$ is smaller than $3 / 5$, resulting in the slope smaller than $-1 / 4$. At present, there are no theories to predict quantitatively the $C$ dependence of $\left\langle r^{2}\right\rangle$ of such low molecular weight polymers.

Acknowledgements. This work was supported in part by The Institute of Polymer Research, Osaka University.

\section{REFERENCES}

1. W. H. Stockmayer, Pure Appl. Chem., 15, 539 (1967).

2. K. Adachi and T. Kotaka, Macromolecules, 16, 1936 
(1983).

3. K. Adachi and T. Kotaka, Macromolecules, 17, 120 (1984).

4. K. Adachi and T. Kotaka, Macromolecules, 18, 294 (1985).

5. K. Adachi and T. Kotaka, Macromolecules, 18, 466 (1985).

6. K. Adachi, H. Okazaki, and T. Kotaka, Macromolecules, 18, 1486 (1985).

7. K. Adachi, H. Okazaki, and T. Kotaka, Macromolecules, 18, 1687 (1985).

8. M. E. Baur and W. H. Stockmayer, J. Chem. Phys., 43, 4319 (1965).

9. W. H. Stockmayer and J. J. Burke, Macromolecules, 2, 647 (1967).

10. A. A. Jones, W. H. Stockmayer, and R. J. Molinari, J. Polym. Sci., Polym. Symp., No. 54, 227 (1976).

11. A. M. North and P. J. Phillips, Trans. Faraday Soc., 64, 3235 (1968).

12. M. Hirose, N. Yamakawa, K. Araki, and Y. Imamura, Rep. Prog. Polym. Phys. Jpn., 20, 117 (1977).

13. S. Mashimo, S. Yagihara, and A. Chiba, Macromolecules, 17, 630 (1984).

14. P. E. Rouse, J. Chem. Phys., 21, 1272 (1953).

15. B. H. Zimm, J. Chem. Phys., 24, 269 (1956).

16. P.-G. de Gennes, J. Chem. Phys., 55, 572 (1971).

17. P.-G. de Gennes, "Scaling Concepts. in Polymer Physics," Cornel University Press, Ithaca, New York, 1979.

18. P.-G. de Gennes, Macromolecules, 9, 587, 594 (1976).

19. M. Doi and S. F. Edwards, J. Chem. Soc., Faraday
Trans. 2, 74, 1789, 1802, 1808 (1978)

20. W. H. Stockmayer and M. E. Baur, J. Am. Chem. Soc., 86, 3485 (1964).

21. H. Yamakawa, "Modern Theory of Polymer Solutions," Harper and Row, New York, N. Y., 1971.

22. M. Daoud and G. Jannink, J. Phys. (Paris), 37, 973 (1976).

23. S. F. Edwards, Proc. Phys. Soc. (London), 88, 265 (1966).

24. S. F. Edwards and E. F. Jeffers, J. Chem. Soc., Faraday Trans. 2, 75, 1020 (1979).

25. A. M. Donald and E. J. Kramer, Polymer, 23, 457 (1982).

26. W. W. Graessley, Adv. Polym. Sci., 16, 1 (1974).

27. J. D. Ferry, "Viscoelastic Properties of Polymers," Wiley, New York, N. Y., 1961.

28. R. S. Moore and J. D. Ferry, J. Phys. Chem., 66, 2699 (1962).

29. P.-G. de Gennes, J. Phys. (Paris), 36, 1199 (1975).

30. M. Doi and N. Y. Kuzuu, J. Polym. Sci., Polym. Lett. Ed., 18, 775 (1980).

31. C. J. F. Böttcher, "Theory of Electric Polarization," Vol. I, Elsevier, Amsterdam, 1973.

32. M. Daoud, J. P. Cotton, B. Frarnoux, G. Jannink, G. Sarma, H. Benoit, R. Duplessix, C. Picot, and P.G. de Gennes, Macromolecules, 8, 804 (1975).

33. R. W. Richards, A. Maconnachie, and G. Allen, Polymer, 22, 147, 153, 157 (1981).

34. J. S. King, W. Boyer, G. D. Wingnall, and R. Ullmann, Macromolecules, 18, 709 (1985). 